\title{
Effects of Training to Improve Novice Nurses' Sense of Coherence
}

\author{
Fumiko Nakajima', Megu Aoyama², Mari Azuma ${ }^{3}$, Chiharu Akazawa ${ }^{4}$ \\ ${ }^{1}$ Department of Nursing, Sugiyama Jyogakuen University, Nagoya, Japan \\ ${ }^{2}$ Nursing Department, Kyoto Okamoto Memorial Hospital, Kyoto, Japan \\ ${ }^{3}$ Department of Nursing Science, Tenri Health Care University, Nara, Japan \\ ${ }^{4}$ School of Nursing, Osaka Medical and Pharmaceutical University, Osaka, Japan \\ Email: f-nakajima@sugiyama-u.ac.jp
}

How to cite this paper: Nakajima, F., Aoyama, M., Azuma, M. and Akazawa, C. (2022) Effects of Training to Improve Novice Nurses' Sense of Coherence. Health, 14, 281-295.

https://doi.org/10.4236/health.2022.143022

Received: February 2, 2022

Accepted: February 28, 2022

Published: March 3, 2022

Copyright (C) 2022 by author(s) and Scientific Research Publishing Inc. This work is licensed under the Creative Commons Attribution International License (CC BY 4.0).

http://creativecommons.org/licenses/by/4.0/

\begin{abstract}
Background: Our previous research concerned the relation between Sense of Coherence, workplace stress, and burnout for nurses in their first three years of work. We found that their psychological state in the first three months on the job was particularly critical to their success and retention. As workplace stress increased over their first three years, their Sense of Coherence declined, and their burnout increased. Follow-up interviews after five years revealed the importance of identifying role models early on and feeling their own growth in the responses of their patients. Based on these findings, in 2015 we instituted a program to improve new nurses' Sense of Coherence. The present research was to evaluate the effectiveness of this training program. Purpose: The purpose of this study was to evaluate the effectiveness of a training program in raising Sense of Coherence. Method: From June of 2015 to March of 2017, we surveyed Sense of Coherence of 137 new nurses who completed our training program, comparing them to a cohort of 960 nurses whom we had studied in 2010-11 without such a training program. We analyzed their responses using SPSS Version 24.0, taking a probability of less than $5 \%$ to be significant. Results: In terms of Sense of Coherence, the training program improved new nurses' "comprehensibility" and "manageability" in the first three months, but both dropped to levels comparable to the untrained nurses after six months. There was no significant difference in "meaningfulness", which dropped over the first year in both groups. Categorized into three groups according to SOC score at the time of employment in the intervention group, 36 participants were classified into the low score group, 41 participants were classified into the medium score group, and 44 participants were classified into the high score group. Then changes in SOC in each group were then compared. In the LOW group, the scores for "comprehensibility", "managea-
\end{abstract}


bility", and "meaningfulness" increased significantly throughout the year, while in the HIGH group, scores decreased significantly. Conclusions: Our training program did raise new nurses' sense of growth over the first year, and significantly improved their "comprehensibility" and "manageability" over the first three months. However, the fact that their Sense of Coherence dropped to control group levels after six months suggests the need for further intervention to maintain "comprehensibility" and "manageability" over the longer term. In the LOW group, the scores for "comprehensibility", "manageability", and "meaningfulness" increased significantly throughout the year, however, the total SOC score for the LOW group remained low throughout the year. Thus, additional support is needed over-and-above the training presented in this study for those nurses with low SOC scores at time of employment.

\section{Keywords}

Novice Nurse, Work Environment, Adaptation, Stress Coping, SOC

\section{Introduction}

In present-day Japan, which is experiencing an increasingly aging population, working conditions for nurses are severe and continue to deteriorate. While the nature of nurses' work is becoming more complex and diverse due to advancements in medical care, nursing also involves a variety of stressors [1]. Among these is burnout resulting from chronic interpersonal stress, which has long been viewed as a problem in terms of inducing nurse turnover and ill health. Many studies on stress and burnout have been conducted and based on their results, measures for stress relief and burnout prevention for nurses have been undertaken [2] [3] [4]. For nurses entering the profession, stress relief is provided through group training, and individual measures are employed when poor adaptation to the workplace becomes apparent. However, in today's medical landscape, where stress is increasing along with advancements in medical care, it is necessary to provide not only measures to relieve stress and prevent burnout but also to focus on providing measures that improve a nurse's ability to cope with stress.

Stress varies in magnitude depending on an individual's capacity to cope with said stress, which is referred to as a "Sense of Coherence" (SOC). An individual's SOC consists of three components: 1) comprehensibility, 2) manageability, and 3) meaningfulness. It has been found that among youth, the ability of SOC is enhanced by the stress they are given [5]. Therefore, in the current study, we focused on the SOC of individual nurses and engaged in developing support mechanisms to better encourage novice nurses to adapt to the (stressful) workplace. The first step was to undertake a large-scale longitudinal survey focused on novice nurses, where SOC, burnout, and occupational stress were measured by 
tracking participants throughout various periods of employment, including three month-, six month-, one year-, two year-, and three year-intervals after employment. Results showed that nurses' SOC decreased, and burnout increased during the three-year period when they were working under highly stressful conditions. Furthermore, the psychological burden of their employment was found to be particularly high during the first three months after employment [6].

The second step was to conduct qualitative interviews with participants as part of a follow-up tracing study. Results of these interviews-which were conducted when participants were in their fifth year of employment-showed that participants adapted to their profession despite continuing to work in a harsh internal environment, as they began to perceive their own growth as a result of their patients' reactions to the nursing care they provided. Many nurses had also begun modeling the nursing practices of senior nursing staff around them [7]. Based on these results, the "Education Program to Enhance the SOC of Novice Nurses" was developed and implemented from 2015 to 2016.

This study aimed to clarify the effects of this training program, by comparing the changes in the SOC of novice nurses during a one-year period before and after the program's implementation.

\section{Methods}

\section{1) Research design}

This study consisted of a quantitative research design in which the effect of the intervention presented through implementation of the "Education Program to Enhance the SOC of Novice Nurses" was ascertained by measuring changes in the SOC and burnout of novice nurses during a one-year period. Specifically, a quantitative questionnaire was employed to gather relevant data.

2) Research period

This study was conducted between June 2015 and March 2017.

3) Participants

Participants included 137 nurses who: 1) were newly hired during 2015 and 2016 at hospitals across the Kinki region (these hospitals formed the study's research partners and allowed their novice nurses to undertake the "Education Program to Enhance the SOC of Novice Nurses"; and 2) received the program's relevant training between June 2015 to March 2016.

4) Outline of the "Education Program to Enhance SOC of Novice Nurses"

The outline of the "Education Program to Enhance SOC of Novice Nurses" was built on Stress Care Program, and it was prepared based on the theory of health generation, with the principal investigator in charge.

This education program was conducted three consecutive times in a given year. Training sessions occurred at three, six, and 12 months after employment. The first part of the training required participants to write and reflect on the daily tasks performed by novice nurses and apply these tasks to the components of SOC (i.e., comprehensibility and manageability). Through this process, partici- 
pating nurses focused on their own growth.

Subsequently, by sharing what they had written regarding their tasks with a group of four or five other novice nurses, participants were able to identify growth they had not previously recognized in themselves. In addition, the significance of nursing care that exists within the practices described in "comprehensibility" and "manageability" were applied to "meaningfulness" to give significance to the tasks the nurses performed. By gradually discovering and accumulating the significance of nursing through these processes, the program aimed to assist novice nurses to be better equipped to feel and experience their growth over time. Furthermore, support from senior nurses was positioned throughout this study as a resource to help novice nurses to cope with stress, and group work was carried out with the facilitation of senior nurses [8].

5) Research content

As noted previously, the SOC of novice nurses during a one-year period was surveyed longitudinally using a quantitative questionnaire. SOC consists of three components: 1) comprehensibility, 2) manageability, and 3) meaningfulness, and each element is included in the scale about one-third. The Japanese version of the SOC13 item scale developed by Yamazaki et al. asks for seven levels of answers to answers with different expressions for all questions, and the higher the total score of the answers, the higher the ability to deal with stress. (13-item reduced version; Yamazaki) [9].

6) Ethical Considerations

Since this study was longitudinal in nature, it was necessary to combine the results of all four questionnaires presented in the SOC Evaluation Scale. In order to obtain longitudinal data, data were managed by numbering each research cooperators based on the registry of each research collaborating institution. The researchers numbered these questionnaires in advance and distributed them to participants at the time of the implementation of the program. The completed questionnaires were jointly collected by mail from each hospital.

This study was conducted with the approval of the ethics committee of the university with which this study's research representative is affiliated (No. 145).

7) Analytical method

In order to evaluate the effectiveness of the training developed in this study, two points presented in the following subsections were analyzed. Specifically, data were statistically processed using IBM SPSS Statistics Ver. 24.0, and a significance level of $\mathrm{p}<0.05$ was used for both analyses.

Comparison of changes in SOC before and after training during a oneyear period

1) The results of the intervention group of this study were compared with the results of a longitudinal SOC survey $(n=960)$ conducted in 2010 , which was used as a control group. The same questionnaire was used for the 2010 study which used novice nurses as participants [6]. The changes in mean scores from the first to the fourth surveys of each scale score were then compared using an analysis of variance. Differences in scores between the intervention and control 
groups 12 months post-employment were compared using a t-test.

2) Relationship between the SOC score levels at the time of employment and the effect of training in the intervention group

Participants of this study were classified into three groups: 1) high scores, 2) medium scores, and 3) low scores, as per participants' total SOC score at time of employment. Changes in their mean SOC scores from the first to the fourth surveys were then compared using the analysis of variance. The differences in scores for each group at 12 months post-employment were analyzed using a ttest.

\section{Results}

1) Participants' Attributes (Table 1)

Questionnaires were distributed to 137 novice nurses who received the corresponding training in 2015 and 2016. Of these, 123 (89.8\%) nurse responses (i.e., questionnaires with no data missing throughout the study period) were analyzed.

This study's final participants consisted of 108 women and 15 men, with a mean age of 23.2 years. In terms of professional education, 56 (45.5\%) had graduated from university, $46(37.4 \%)$ had graduated from a vocational school (e.g., registered nurse), and 14 (11.4\%) had undergone a non-degree graduate program (e.g., five-year high school). Qualifications of participants included 118 nurses (95.9\%), three practical nurses (2.4\%), and two public health nurses (1.6\%). In the control group, $634(66.0 \%)$ participants had graduated from vocational schools (e.g., registered nurse), representing the most common educational background, and 136 (14.1\%) had graduated from universities. These findings indicated that that basic nursing education had shifted to primarily 4-year university courses.

2) Comparison of changes in SOC before and after training during a one-year period

Table 1. Participants' attributes.

\begin{tabular}{|c|c|c|}
\hline & participants (123) & control (960) \\
\hline \multirow{2}{*}{ Sex } & Women: 108 (87.8\%) & Women: 864 (90.0\%) \\
\hline & Men: $15(12.2 \%)$ & Men: $96(10.0 \%)$ \\
\hline Age & 23.2 & 25.7 \\
\hline \multirow{3}{*}{$\begin{array}{l}\text { In terms of } \\
\text { Professional } \\
\text { education }\end{array}$} & University: 56 (45.5\%) & Vocational school: 634 (66.0\%) \\
\hline & Vocational: school46 (37.4\%) & University: 136 (14.1\%) \\
\hline & Five-year high school: 14 (11.4\%) & Five-year high school: 94 (9.8\%) \\
\hline \multirow{3}{*}{ Eligibility } & Registered Nurse: 118 (95.9\%) & Registered Nurse: 876 (91.3\%) \\
\hline & Associate Nurse: 3 (2.4\%) & Associate Nurse: 43 (4.5\%) \\
\hline & Public Health Nurse: 2 (1.6\%) & Public Health Nurse: 33 (3.4\%) \\
\hline
\end{tabular}


The changes in SOC during the one-year period after the initial employment of the intervention group (i.e., those participants who took the "Education Program to Enhance the SOC of Novice Nurses") was compared with the survey result of the control group, who had carried out a similar questionnaire in 2010.

SOC consists of sub-concepts such as "comprehensibility" (i.e., ability to understand what is happening), "manageability" (i.e., ability to manage the situation), and "meaningfulness" (i.e., feeling that our lives have some kind of emotional meaning). Having higher scores in all three concepts signifies that one has a stronger ability to cope with stress [9]. The transition of the mean scores from the first to the fourth surveys of each sub-concept of the SOC scale was then compared.

First, it was found that "comprehensibility" significantly increased from 17.6 at the time of employment to 18.7, three months later in the intervention group, which greatly differed from the control group. In the control group, this factor significantly decreased from 18.2 to 17.8 , respectively. Scores on each survey from the time of employment to 12 months later were significantly higher in the intervention group at three months post-employment; however, there were no significant differences in other scores (Figure 1).

Next, "manageability" was found to have increased in the intervention group from 16.4 at the time of employment to 17.0, three months later, although no significant difference was found. This finding differed from the significant decrease seen in the control group (i.e., from 16.8 to 16.5 , respectively). After three months onwards, however, the scores of the intervention group began to decrease, which indicated similar changes as those present within the control group. There were also no significant differences across the scores pertaining to each survey between the two groups (Figure 2).

Third, "meaningfulness" decreased in the intervention group from 18.7 at the time of employment to $18.3,17.5$, and 17.4 at three, six, and 12 months later, respectively. These findings revealed that there was a decrease throughout the

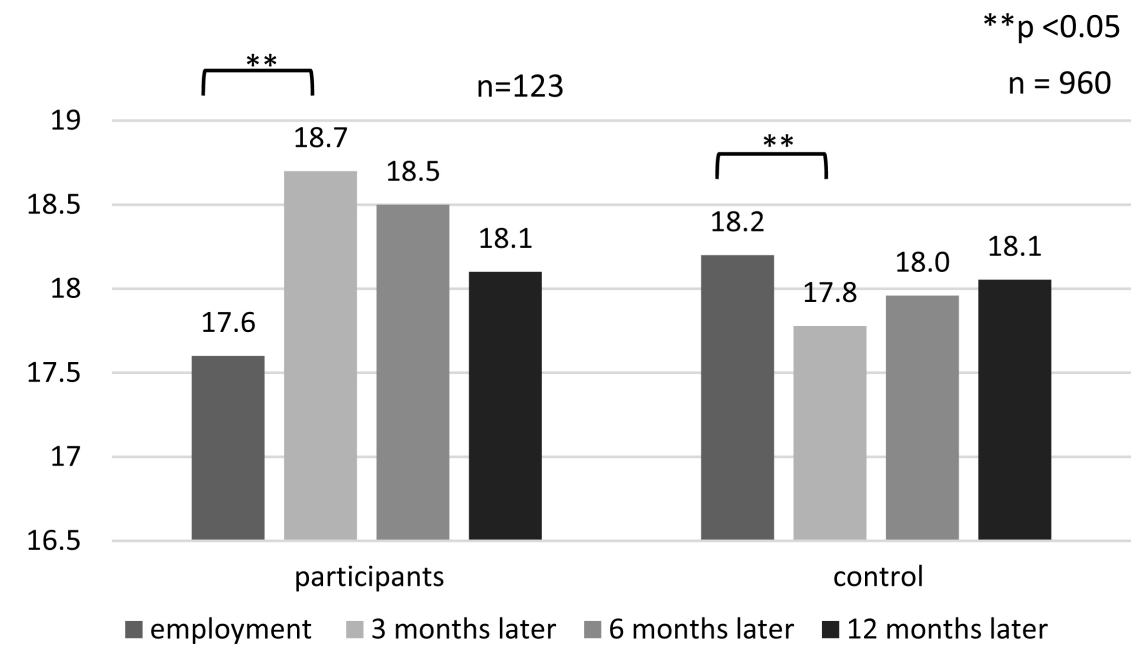

Figure 1. Changes of comprehensibility. 


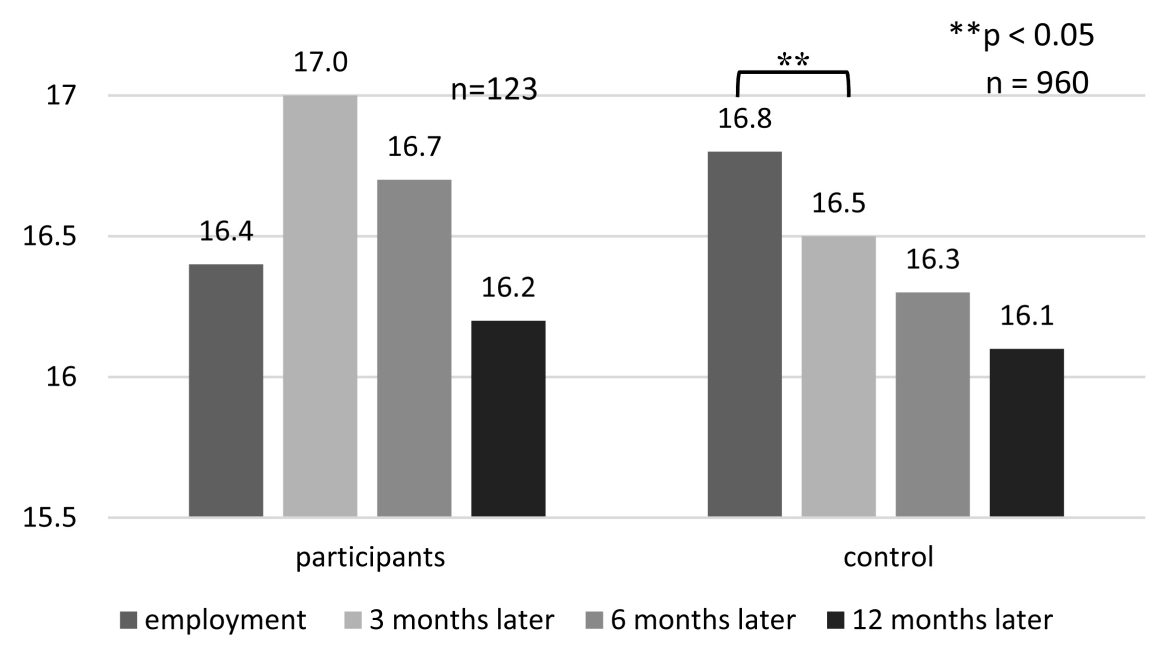

Figure 2. Changes of manageability.

year in scores, although there was no significant difference. In comparison to the control group, the trend of decline was found to be more gradual in the intervention group than in the control group. In the control group, each survey showed a significant decline, from 19.6 at the time of employment to 18.3, 18.1, and 17.8 at three, six, and 12 months later. For the scores of each survey between the two groups, it was found that the intervention group scored significantly lower at time of employment. However, there was no significant difference in subsequent scores between the two groups (Figure 3).

Finally, in the intervention group, participants' total SOC score increased from 52.7 at the time of employment to 54.0, three months later. Although there was no significant difference, the score showed an increase, then began to decrease. This was significantly different in the control group, where participants' total SOC score significantly decreased from 54.5 at time of employment to 52.6, three months later. There were, however, no significant differences found in the scores across each survey between the two groups (Figure 4).

3) Relationship between SOC score levels at the time of employment and the effect of training in the intervention group

Based on the total SOC score at the time of employment in the intervention group, 36 participants with $29-47$ points were classified into the low score group (hereinafter referred to as the "LOW group"), 41 participants with 48 - 55 points were classified into the medium score group (hereinafter referred to as the "MED group"), and 44 participants with 56 - 76 points were classified into the high score group (hereinafter referred to as the "HIGH group"). This classification was done so that each group had approximately the same number of participants. The changes in SOC in each group were then compared.

In the LOW group, the mean scores for "comprehensibility," "manageability," and "meaningfulness" increased from the time of employment to three months later, decreased once over six months, increased again over 12 months, and increased significantly over 12 months after employment. 


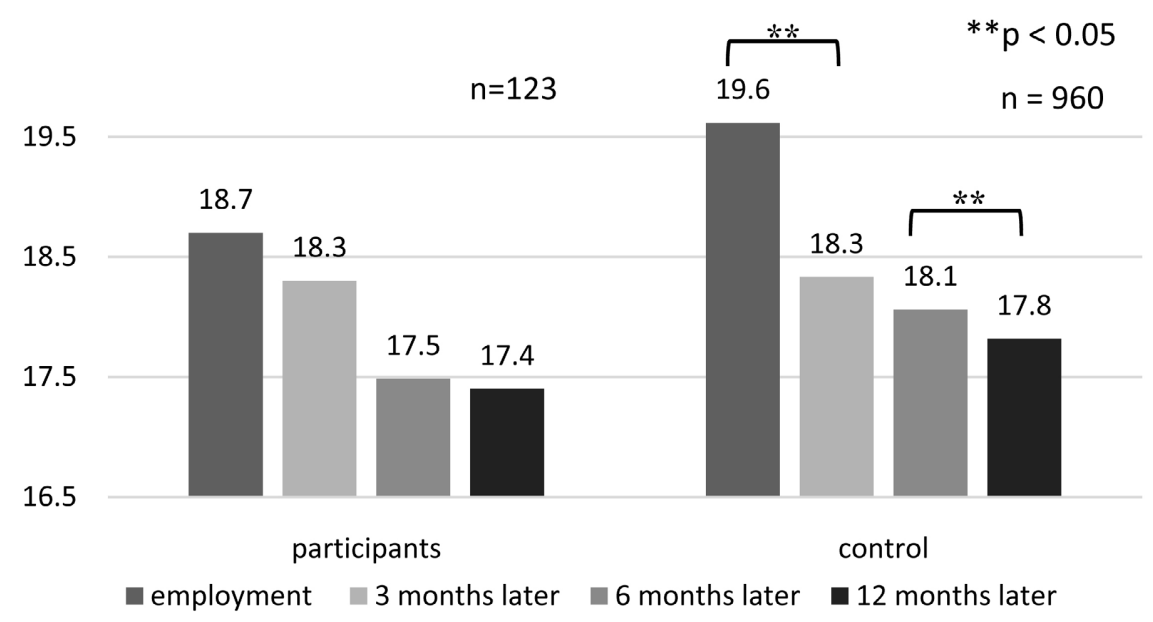

Figure 3. Changes of meaningfulness.

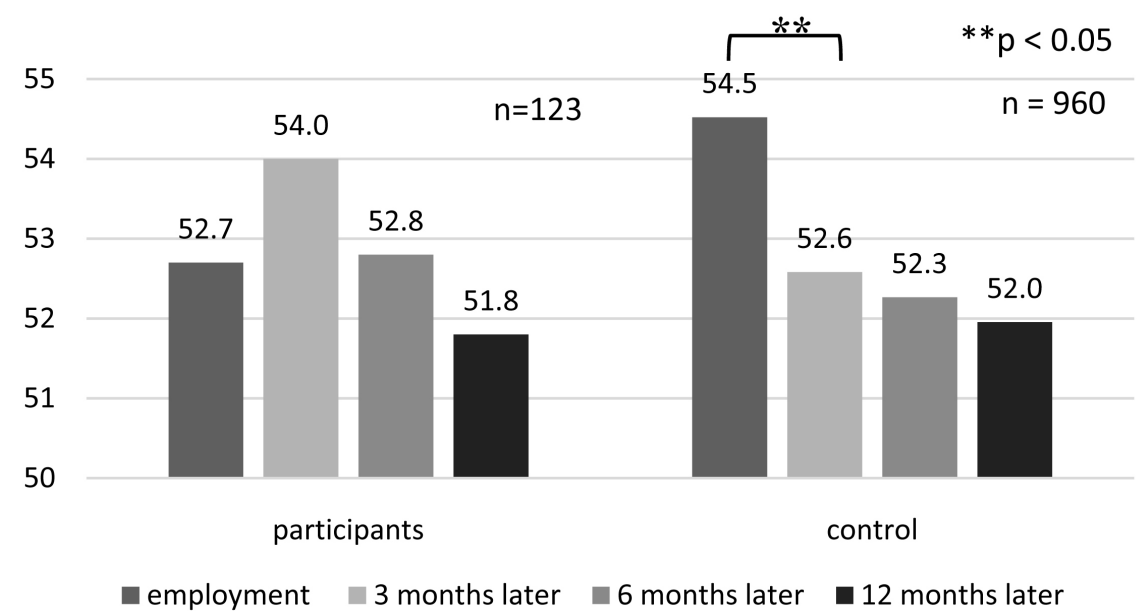

Figure 4. Changes of total SOC score.

In the MED group, the mean scores for "comprehensibility," "manageability," and "meaningfulness" all increased slightly after three months of employment and then fluctuated slightly thereafter. However, there was no significant difference found throughout the year.

In the HIGH group, the mean scores for "comprehensibility," "manageability," and "meaningfulness" all decreased after three months of employment, increased once during the six-month period, decreased again during the 12-month period, and decreased significantly after 12 months of employment (Figures 5-7).

In terms of the total SOC score, which consisted of the sum of each scale, the total score from time of employment to 12 months later significantly increased in the LOW group, significantly decreased in the HIGH group, and did not significantly change in the MED group (Figure 8).

Both the scores of "comprehensibility" and "manageability" in each group were found to be higher at three months and six months after employment for all three groups (LOW, MED, and HIGH, chronologically), with a significant 


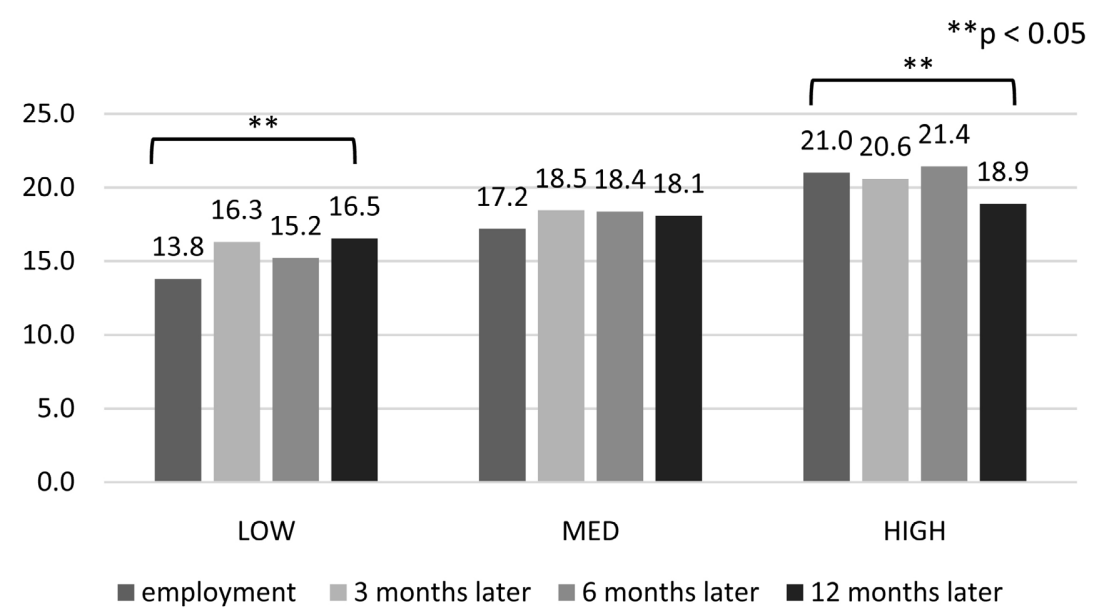

Figure 5. Changes in the comprehensibility of each group.

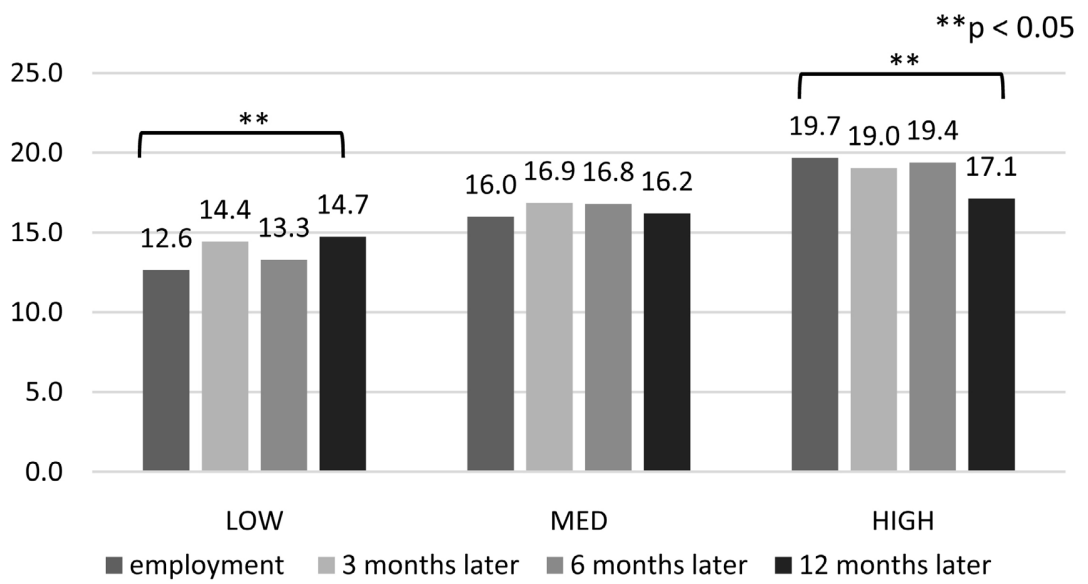

Figure 6. Changes in the manageability of each group.

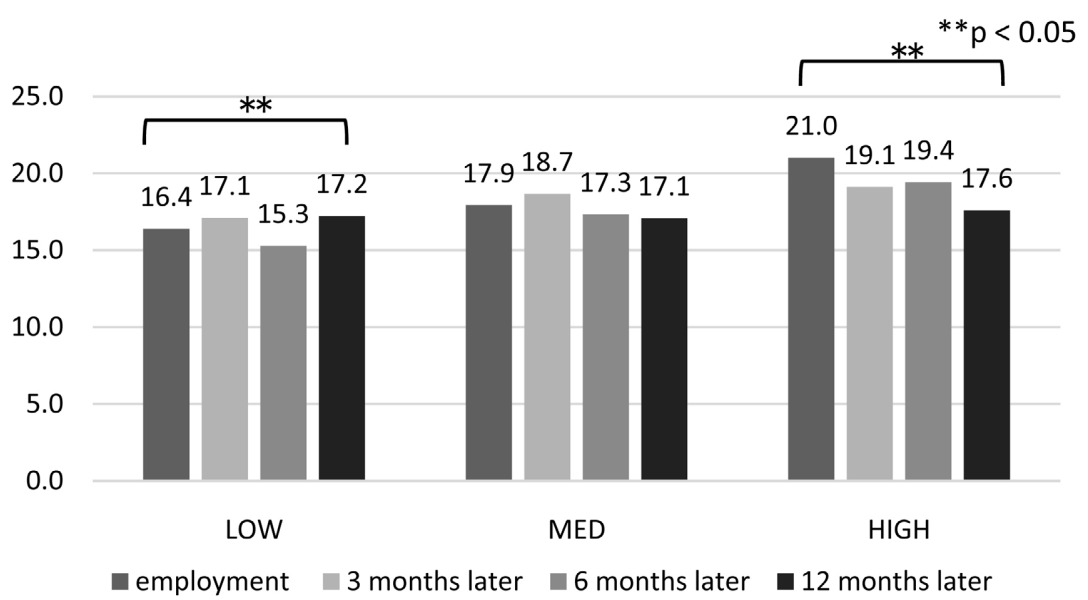

Figure 7. Changes in the meaningfulness of each group.

difference identified. However, at 12 months post-employment, only the LOW and HIGH groups were found to have a significant difference, with the HIGH group having the higher of the two scores (Table 2 and Table 3 ). 
Table 2. Comparison of comprehensibility by group.

\begin{tabular}{|c|c|c|c|c|c|c|c|c|}
\hline & employment & & 3 months later & & 6 months later & & 12 months later & \\
\hline LOW $(\mathrm{n}=36)$ & $13.8 \pm 2.4$ & & $16.3 \pm 3.3$ & & $15.2 \pm 3.8$ & & $16.5 \pm 3.2$ & \\
\hline $\operatorname{MED}(\mathrm{n}=41)$ & $17.2 \pm 2.6$ & & $18.5 \pm 3.4$ & & $18.4 \pm 3.8$ & & $18.1 \pm 4.3$ & ** \\
\hline HIGH $(\mathrm{n}=44)$ & $21.0 \pm 3.5$ & ** & $20.6 \pm 3.6$ & ** & $21.4 \pm 2.7$ & $* *$ & $18.9 \pm 3.8$ & \\
\hline
\end{tabular}

$\mathrm{p}<0.01^{* *}$

Table 3. Comparison of manageability by group.

\begin{tabular}{|c|c|c|c|c|c|c|c|c|}
\hline & employment & & 3 months later & & 6 months later & & 12 months later & \\
\hline LOW $(\mathrm{n}=36)$ & $12.6 \pm 2.3$ & ]$_{* *}$ & $14.4 \pm 3.4$ & ]$_{* *}$ & $13.3 \pm 3.5$ & ** & $14.7 \pm 3.8$ & \\
\hline $\operatorname{MED}(\mathrm{n}=41)$ & $16.0 \pm 2.1$ & & $16.9 \pm 2.8$ & & $16.8 \pm 3.5$ & & $16.2 \pm 3.7$ & ** \\
\hline $\mathrm{HIGH}(\mathrm{n}=44)$ & $19.7 \pm 2.9$ & $\int * *$ & $19.0 \pm 3.2$ & $\int * *$ & $19.4 \pm 3.4$ & $* *$ & $17.1 \pm 3.7$ & \\
\hline
\end{tabular}

$\mathrm{p}<0.01^{* *}$.

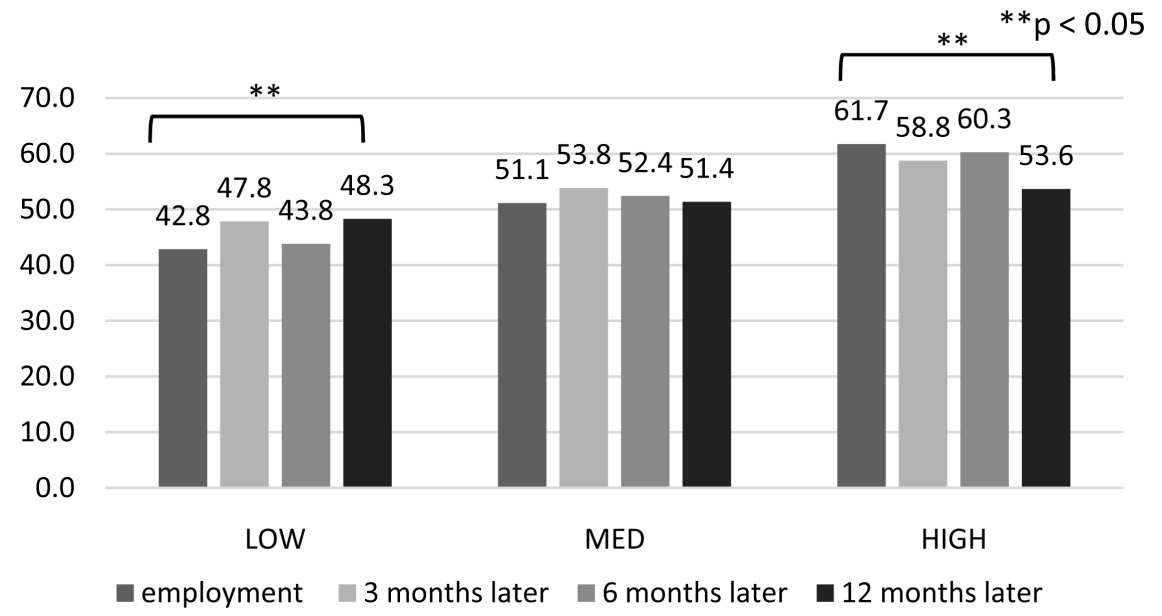

Figure 8. Changes in the total SOC score of each group.

In terms of the "meaningfulness" scores for each group, significant differences were found between all three groups (i.e., LOW, MED, and HIGH) at time of employment as well as six months later, as well as between the LOW and HIGH groups at three months after employment. After 12 months, however, there were no significant differences between the groups' scores (Table 4). The total SOC scores had a similar result to those of "comprehensibility" and "manageability" (Table 5).

\section{Discussion}

In this study, the effect of the "Education Program to Enhance the SOC of Novice Nurses" was measured by comparing changes in the SOC of novice nurses before and after the introduction of the program over a one-year period. Additionally, the effect of the training by participant was examined by comparing the 
Table 4. Comparison of meaningfulness by group.

\begin{tabular}{|c|c|c|c|c|c|c|c|}
\hline & employment & & 3 months later & & 6 months later & & 12 months later \\
\hline $\operatorname{LOW}(\mathrm{n}=36)$ & $16.4 \pm 3.6$ & 7 & $17.1 \pm 3.8$ & & $15.3 \pm 3.9$ & & $17.2 \pm 3.2$ \\
\hline $\operatorname{MED}(\mathrm{n}=41)$ & $17.9 \pm 3.3$ & * & $18.7 \pm 3.1$ & $* *$ & $17.3 \pm 3.6$ & & $17.1 \pm 3.8$ \\
\hline $\mathrm{HIGH}(\mathrm{n}=44)$ & $21.0 \pm 2.8$ & & $19.1 \pm 3.4$ & & $19.4 \pm 3.3$ & & $17.6 \pm 4.0$ \\
\hline
\end{tabular}

$\mathrm{p}<0.01^{* *}$

Table 5. Comparison of total SOC score by group.

\begin{tabular}{|c|c|c|c|c|c|c|c|c|}
\hline & employment & & 3 months later & & 6 months later & & 12 months later & \\
\hline LOW $(\mathrm{n}=36)$ & $13.8 \pm 2.4$ & & $16.3 \pm 3.3$ & - & $15.2 \pm 3.8$ & & $16.5 \pm 3.2$ & \\
\hline $\operatorname{MED}(\mathrm{n}=41)$ & $17.2 \pm 2.6$ & & $18.5 \pm 3.4$ & & $18.4 \pm 3.8$ & & $18.1 \pm 4.3$ & $* *$ \\
\hline HIGH $(\mathrm{n}=44)$ & $21.0 \pm 3.5$ & ** & $20.6 \pm 3.6$ & $\left.\right|^{* *}$ & $21.4 \pm 2.7$ & ** & $18.9 \pm 3.8$ & \\
\hline
\end{tabular}

$\mathrm{p}<0.01^{\star *}$

relationship between the changes in participants' SOC over a one-year period with their SOC levels at the time of employment. Each result is discussed in further detail in the following sections.

1) Comparison of changes in SOC during a one-year period before and after the training program

The mean total SOC score at participants' time of employment was 52.7 points in the intervention group and 54.5 points in the control group. Compared with the mean SOC score of 51.9 points for nurses surveyed by [10], neither the intervention group nor the control group in the current study exhibited low SOC levels. Participants' scores in "comprehensibility" also increased significantly in the intervention group between the time of employment to three months later. The SOC scores were also significantly higher in the intervention group at three months post-employment.

By comparison, participants' scores for "manageability" increased in the intervention group three months after their start of employment, although there was no significant difference in this increase. The total SOC score also increased significantly three months post-employment. Therefore, it is supposed that participants' overall SOC may have increased as a result of an increase in both "comprehensibility" and "manageability". This result was found to be substantially different from the significant decrease in SOC three months post-employment witnessed in the control group [8].

It should be noted that the training functioned by having nurses reflect on their daily nursing practices in order to discover what they had come to understand and what they had become able to do. Subsequently, they experienced growth by receiving positive feedback in this regard. Karasawa et al. previously reported that, based on a survey of novice nurses one and three months after employment, nurses experienced difficulty when they felt that they were not al- 
lowed to wait for their growth because they had to treat patients who were right in front of them [11]. However, the training program developed in this study led to a reduction in the sense of difficulty unique to novice nurses. As a result, by the time they had been employed for three months, a period in which novice nurses experience the most stress and decreased SOC, participating nurses in this study were believed to have increased their SOC by feelings stated as "I have been able to grow to this point" and "I think even I can do it".

However, after six months, the scores for "comprehensibility", "manageability", and total SOC began to decline, which suggested the need for further measures to maintain SOC once it had been raised. Since this training consisted of group training, it offered an opportunity for participants to look closely and objectively at themselves, away from daily work. Currently, however, on-the-job (OJT) personnel, such as preceptors and partners, are not being taught about education policies and approaches toward novice nurses. Tomoyasu et al. further reported that leader-related factors that novice nurses tended most to critique included "poor approachability" (e.g., being difficult to talk to) and "emotional attitudes" (e.g., being harsh and irritable) [12]. It is necessary, therefore, to better coordinate with OJT staff in the future, so that when novice nurses return to daily work, they do not feel as though the growth they experienced during the group training sessions is futile due to the poor attitudes of OJT leaders and/or by being severely criticized by OJT leaders for their inexperience.

With regard to "meaningfulness", scores continuously decreased over the 12 month period in the control group (i.e., from time of employment to completion of the study). The score similarly decreased in the intervention group during the 12-month period, despite there being no significant difference. "Meaningfulness" is an important feeling that leads to motivation and staying power when facing new challenges. Thus, the findings indicate that it is necessary to devise ways to enhance "meaningfulness" so that novice nurses can perceive daily experiences as meaningful.

2) Relationship between SOC score levels at the time of employment and the effect of training in the intervention group

In the LOW group, the scores for "comprehensibility", "manageability", and "meaningfulness" increased significantly throughout the year (despite fluctuation), while in the HIGH group, scores decreased significantly, resulting in a similar total SOC score change. Based on these findings, it can be expected that novice nurses with low SOC at the time of employment are likely to improve their SOC to some extent through training. However, the total SOC score for the LOW group was 42.8 at the time of employment and 48.3 at 12 months postemployment. Despite the increased score, it was still lower than the mean SOC score of 51.9 for nurses surveyed by Yoshida et al. [10]. Thus, additional support is needed over-and-above the training presented in this study for those nurses with low SOC scores at time of employment.

Conversely, the mean total SOC score in the HIGH group was 61.7 at time of 
employment and 53.6 at 12 months post-employment-both of which were higher scores than the mean SOC score of nurses surveyed by Yoshida et al. [10]. This finding remains true even after accounting for the current study's decrease in participants' scores. In addition, the total SOC score in the MED group was 51.1 at time of employment and 51.4 at 12 months post-employment. These results are similar to findings by Yoshida et al. [10]. There was also no significant change throughout the 12-month period for this group.

Based on the presented findings, it is believed that novice nurses with high SOC scores at time of employment, as well as novice nurses that present with average SOC scores (regardless of whether they receive training or not), both possess high abilities for dealing with stress and can cope effectively on their own. However, in the HIGH group, findings also indicated a decline in the natural abilities of these nurses to cope with stress throughout the year. This finding implies that the situation in which novice nurses find themselves over time can be severely stressful. It is believed, therefore, that conventional support that promotes stress relief could be effective for these participants.

Recognizing the differences between the LOW and HIGH groups when comparing scores by group would naturally occur, as participants were classified into three groups and then compared according to the overall SOC score. However, it should be noted that although the mean score of "meaningfulness" was significantly higher in the HIGH group than in the LOW group between time of employment and six months later, there was no significant difference at 12 months post-employment. It is essential, therefore, to encourage nurses to improve their meaningfulness, regardless of their SOC at time of employment, as meaningfulness could significantly serve as motivation when faced with difficult life events.

Furthermore, both "comprehensibility" and "manageability" were found to be easily visualized as something relevant to participants by having them concretely recall their daily practices during the training program. "Meaningfulness", in turn, required that practice content be related to both participants' knowledge and theory, and for participants' experiences to be verbalized. Training facilitators in this study indicated that it is often difficult for novice nurses to translate their experiences into language when filling outgrowth confirmation sheets or performing group work, and that it is common to see novice nurses struggling with support. We believe that by examining the relationship between these characteristics and SOC, it may be possible to develop new support measures for novice nurses with low SOC at the time of employment (i.e., nurses that require more support).

\section{Conclusions}

In this study, an educational program aimed at enhancing the SOC of novice nurses was developed and evaluated by focusing on nurses' SOC. Results indicated that although the program increased participants' SOC during periods of high stress (i.e., three months post-employment), their SOC decreased again af- 
ter six months. Therefore, it is necessary to develop further measures to maintain the effectiveness of the presented training over time.

In addition, although novice nurses with low SOC at the time of employment were found to benefit from the intervention in terms of "comprehensibility" and "manageability", their SOC remained low throughout the year. Therefore, new measures are needed to better enhance "meaningfulness" for those with low SOC.

\section{Limitations of This Study}

Although a certain degree of positive effect was recognized in the education program developed for this study, there is room for improvement in order to better maintain the noted effect. Further improvement can also be gained by preparation of a manual, which could allow anyone to conduct the same kind of intentional positive feedback given by the lecturer during the training as well as conduct practice methods for ascribing significance to clinical practice. This manual could also be provided to more participants in the future.

\section{Acknowledgements}

We would like to extend our heartfelt gratitude to all our participants as well as the nursing administrators of collaborating hospitals who made this study possible.

\section{Fund}

This study was funded by the 2013 JSPS KAKENHI Grant-in-Aid for Challenging Exploratory Research.

\section{Conflicts of Interest}

The authors declare no conflicts of interest regarding the publication of this paper.

\section{References}

[1] Toshiko, I. (1984) Analyzing the Stress of Clinical Nurses. The Japanese Journal of Nursing, 48, 999-1005.

[2] Ritsuko, Y. (2005) Why Do Nurses Burnout? Metal Health Measures Learned from Anti-Nurse Burnout Programs in North America. Japanese Journal of Nursing, 69, 228-232.

[3] Fumiaki, I. (1990) Realities, Factors, and Prevention of the Burnout of Nurses. Journal of Clinical and Experimental Medicine, 153, 243-246.

[4] Masae, M., Yasuko, S. and Akihiro, S. (2010) Research on Factors Related to Novice Nurses' Workplace Adaptation. The Journal of Japan Academy of Health Sciences, 12, 211-220.

[5] Antonovsky, A., Yoshihiko, Y. and Kiyoko, Y. (Trans.) (2001) Solving the Mysteries of Health: Mechanism of Stress Coping Abilities and Health Management. Yushindo, Tokyo. 
[6] Fumiko, N. (2017) Support for Novice Nurses to Adapt to Workplaces: Things That Become Support for Overcoming Difficulties. Kyoto University Human and Environmental Studies Doctoral Thesis.

[7] Fumiko, N. (2017) Process of the Workplace Adaptation of Novice Nurses: Analysis of Accounts of Fifth-Year Nurses. Nursing Research of Sugiyama Jogakuen University, 9, 1-13.

[8] Fumiko, N. (2017) Realities of an Education/Training Program Utilizing SOC. Nursing Resource Development, 14, 53-60.

[9] Yoshihiko, Y. (1999) Theorist Who Adopted a New Approach to Health: Salutogenesis and the Sense of Coherence (SOC). Quality Nursing, 5, 81-88.

[10] Eri, Y., Kazuko, Y., Hiromi, S., et al. (2013) Correlation between Nurses' Sense of Coherence and Stress Reaction. Japanese Journal of Nursing Research, 36, 25-33.

[11] Yumiko, K., Megumi, N., Hiroko, H., et al. (2010) Work Difficulties and Support Desired Felt by Novice Nurses at One Month and Three Months after Employment. Journal of Japan Academy of Health Sciences, 12, 211-220.

[12] Hideki, T., Tetsuharu, I., Michiko, T., et al. (2015) State of Newcomer Education from the Perspective of Novice Nurses: Examination into an Effective Education System. Japanese Psychiatric Nursing Society, 58, 156-160. 